\title{
Challenges to be addressed in management of patients with sinusoidal obstruction syndrome after hematopoietic cell transplantation
}

Seong Kyu Park, Young Sok Ji, and Se Hyung Kim

Division of Hematooncology, Department of Internal Medicine, Soonchunhyang University Bucheon Hospital, Bucheon, Korea

Received : November 30, 2021

Accepted: December 21, 2021

\section{Correspondence to}

Seong Kyu Park, M.D.

Division of Hematooncology,

Department of Internal Medicine,

Soonchunhyang University Bucheon

Hospital, 170 Jomaru-ro, Wonmi-gu,

Bucheon 14584, Korea

Tel: +82-32-621-5185

Fax: +82-32-621-5018

E-mail: skpark@schmc.ac.kr https://orcid.org/0000-0002-30553621

\section{See Article on Page 179-189}

Hepatic sinusoidal obstruction syndrome (SOS; previously known as venoocclusive disease) is a lethal complication that typically presents within days or weeks after hematopoietic cell transplantation (HCT) with refractory thrombocytopenia, hepatomegaly, ascites, and jaundice. Although SOS is not common, it can progress rapidly to multiorgan dysfunction and death. Therefore, attention and close monitoring are needed for early diagnosis of SOS, and early management is essential to reduce the associated morbidity and mortality.

Similar to other HCT-related complications, several challenges must be addressed in terms of the diagnosis and management of SOS, including lack of universally accepted diagnostic and severity grading criteria, the appropriate timing and scope of defibrotide administration, identification of patients at high risk based on clinical factors, and the prophylactic use of defibrotide.

Diagnostic and severity grading criteria have been revised several times since the 1980s. Multiple risk factors for SOS have been identified, but often confused with other hepatic complications due to the nonspecific clinical features. The Baltimore [1] and modified Seattle criteria [2] were widely used in the past, but some patients developed SOS despite not meeting these classical criteria. These patients, particularly pediatric patients or patients with late-onset SOS, had normal bilirubin levels $[3,4]$. The European Society for Blood and Marrow Transplantation (EBMT) suggested revised diagnostic criteria [5] in 2016 that divided SOS into classic SOS $(\leq 21$ days after HCT) and late-onset SOS (> 21 days after HCT).

In the issue of the Korean Journal of Internal Medicine on SOS, Kong et al. [6] reported that the diagnostic criteria affected the treatment outcomes. Patients with hyperbilirubinemia (>2 $\mathrm{mg} / \mathrm{dL}$ ) included as a prerequisite for the Baltimore criteria had poorer survival rates than those meeting the modified Seattle criteria. This was thought to be because cases that met the Baltimore criteria had more severe sos compared to those meeting the modified Seattle criteria. In this study, the two classical diagnostic criteria were used, but analysis using the revised EBMT criteria was not performed. It is predicted that the diagnostic criteria will also affect the overall incidence evaluation of SOS. According to the data published by Yoon et al. [7], the overall incidence of SOS ranged from $7.8 \%$ to $8.9 \%$ of all transplants. This incidence was lower than that reported in other large-scale studies (14\% to $15 \%$ ). 
The incidence of SOS recently reported by Yoon et al. [8] was 3.4\% after allogeneic hematopoietic stem cell transplantation, which was a very low value considering that half of the patients received myeloablative conditioning. They used the usual prophylactic strategy of oral ursodiol and intravenous heparin or prostaglandin E1. The prophylactic effects of drugs other than ursodiol have not been clearly proven. The frequency reported in Korea is generally low. Although the reason for the difference is unknown, it may be because the diagnostic criteria applied at each institution are different.

In addition, the authors reported that creatinine levels affected the survival rate in SOS patients treated with defibrotide. Cairo et al. [9] reported a similar result. They recently developed further revised diagnostic criteria for SOS that emphasize the importance of early diagnosis using general clinical parameters and dysfunction of organs other than the liver (i.e., kidney, lung, heart, and central nervous system). As renal dysfunction has been introduced into the new EBMT severity grading criteria, a validation process is needed to determine whether it can be applied to actual clinical practice in Korea. Recently, one institution reported the results of a validation study of the EBMT severity grading criteria, and is expected to be a good reference [10].

Similar to the difference in incidence of SOS in related reports in Korea, there are also differences in the distribution of severity [10]. These differences are also thought to be due to the use of different criteria for SOS grading between institutions. It will be crucial to standardize these criteria for the establishment of a treatment strategy and to determine when to start treatment.

As about 200 cases of SOS occur after hematopoietic stem cell transplantation in Korea each year [7], only a limited number of subjects were analyzed over a long period of more than 18 years in this study. As a small-scale study, the analytical power was limited. Therefore, efforts are needed to collect and analyze data at the national level.

In this study, there was a significant difference in the survival rate at 100 days according to the severity of SOS in all cases. However, the difference in severity did not significantly affect the survival rate at 100 days in cases treated with defibrotide. These results were considered to confirm the effectiveness of drug treatment. Meanwhile, defibrotide is approved only for severe or very severe SOS. However, defibrotide was administered in mild to moderate cases (51.3\%) outside the indications of this study, which is problematic for interpretation of the results.

In this study, the results were compared between patients who received the drug within 1 day after diagnosis and those who were treated 2 or more days after diagnosis to evaluate the appropriate administration timing of the therapeutic agent. Although not statistically significant, there was a nearly twofold improvement in survival rate when the drug was administered within 1 day after diagnosis. This is consistent with most guidelines, including those of the EBMT, which state that active treatment should be implemented at an early stage through early diagnosis. A multicenter study [11] and a systematic review [12] demonstrated improvement in survival rates only in severe cases receiving defibrotide. In Korea, defibrotide can be reimbursed by the national insurance system only when used in severe or very severe SOS, corresponding to the same conditions under which the drug's effectiveness has been demonstrated in the literature. However, the disease course of SOS is dynamic and progresses rapidly to multiorgan failure within a few days $[7,13]$. Therefore, additional clinical studies are needed to confirm the advantages of early treatment so that active treatment can be applied before progression to severe or very severe disease where the use of defibrotide is currently approved. In addition, it is necessary to define the subgroup in which prophylactic treatment with defibrotide should be actively applied by identifying the clinical characteristics or biomarkers corresponding to high risk.

Given the lack of research on SOS, it is meaningful that the authors analyzed and reported significant prognostic factors related to SOS in this paper. However, a number of issues remain to be addressed with SOS in the future.

\section{Conflict of interest}

No potential conflict of interest relevant to this article was reported.

\section{REFERENCES}

1. Jones RJ, Lee KS, Beschorner WE, et al. Venoocclusive disease of the liver following bone marrow transplantation. Transplantation 1987;44:778-783.

2. McDonald GB, Hinds MS, Fisher LD, et al. Veno-occlusive disease of the liver and multiorgan failure after bone marrow transplantation: a cohort study of 355 patients. Ann Intern 
Med 1993;118:255-267.

3. Mehra V, Tetlow S, Choy A, et al. Early and late-onset veno-occlusive disease/sinusoidal syndrome post allogeneic stem cell transplantation: a real-world UK experience. Am J Transplant 2021;21:864-869.

4. Corbacioglu S, Kernan NA, Pagliuca A, Ryan RJ, Tappe W, Richardson PG. Incidence of anicteric veno-occlusive disease/sinusoidal obstruction syndrome and outcomes with defibrotide following hematopoietic cell transplantation in adult and pediatric patients. Biol Blood Marrow Transplant 2020;26:1342-1349.

5. Mohty M, Malard F, Abecassis M, et al. Revised diagnosis and severity criteria for sinusoidal obstruction syndrome/veno-occlusive disease in adult patients: a new classification from the European Society for Blood and Marrow Transplantation. Bone Marrow Transplant 2016;51:906-912.

6. Kong SG, Lee JH, Lim YT, et al. Influence of creatinine levels on survival in patients with veno-occlusive disease treated with defibrotide. Korean J Intern Med 2022;37:179-189.

7. Yoon $\mathrm{JH}$, Choi CW, Won JH. Hepatic sinusoidal obstruction syndrome/veno-occlusive disease after hematopoietic cell transplantation: historical and current considerations in Korea. Korean J Intern Med 2021;36:1261-1280.

8. Yoon JH, Min GJ, Park SS, et al. Incidence and risk factors of hepatic veno-occlusive disease/sinusoidal obstruction syndrome after allogeneic hematopoietic cell transplantation in adults with prophylactic ursodiol and intravenous heparin or prostaglandin E1. Bone Marrow Transplant 2021;56:16031613.

9. Cairo MS, Cooke KR, Lazarus HM, Chao N. Modified diagnostic criteria, grading classification and newly elucidated pathophysiology of hepatic SOS/NOD after haematopoietic cell transplantation. Br J Haematol 2020;190:822-836.

10. Yoon JH, Yoo KH, Sung KW, et al. Validation of treatment outcomes according to revised severity criteria from European Society for Blood and Marrow Transplantation (EBMT) for sinusoidal obstruction syndrome/veno-occlusive disease (SOS/ VOD). Bone Marrow Transplant 2019;54:1361-1368.

11. Richardson $P G$, Riches $M L$, Kernan $N A$, et al. Phase 3 trial of defibrotide for the treatment of severe veno-occlusive disease and multi-organ failure. Blood 2016;127:1656-1665.

12. Richardson P, Aggarwal S, Topaloglu O, Villa KF, Corbacioglu S. Systematic review of defibrotide studies in the treatment of veno-occlusive disease/sinusoidal obstruction syndrome (VOD/ SOS). Bone Marrow Transplant 2019;54:1951-1962.

13. Carreras E. How I manage sinusoidal obstruction syndrome after haematopoietic cell transplantation. Br J Haematol 2015;168:481-491. 